\title{
The Mechanisms of Mitochondria-Mediated Apoptosis in Cancer Chemotherapy
}

\author{
Ashutosh Shrivastava \\ Division of Experimental Medicine, Beth Israel Deaconess Medical Center, Harvard Medical School, 330 Brookline Ave, Boston, MA 02115
}

Email address:

ashrivas@bidmc.harvard.edu

To cite this article:

Ashutosh Shrivastava. The Mechanisms of Mitochondria-Mediated Apoptosis in Cancer Chemotherapy. Cell Biology. Special Issue: Mitochondria: Implications in Human Health and Diseases. Vol. 3, No. 2-1, 2015, pp. 17-21. doi: 10.11648/j.cb.s.2015030201.13

\begin{abstract}
Cell metabolism and signal transduction has usually been considered independent processes. Interestingly mitochondria is an intracellular organelle that perform energy production to fuel the cell to carry out its normal functions, however, in certain circumstances, its components can also carry out programmed cell destruction via its resident proteins. In this review, the role of mitochondria and its component proteins in inducing apoptotic programmed cell death is discussed. The role of Bcl-2 family proteins that set up the mitochondria induced apoptotic threshold is also described. Further, how the metabolic stress leads to mitochondrial dysfunction via increased generation of reactive oxygen species are discussed. This article is a part of a special issue on mitochondria summarizes the induction of intracellular pathways that converge on mitochondria and ultimately impact the success of chemotherapeutic interventions.
\end{abstract}

Keywords: Mitochondria, Cancer, Apoptosis, Programmed Cell Death

\section{Introduction}

The mitochondrion is an organelle found in higher eukaryotes like plant and animal cells and in lower forms of eukaryotes, such as yeast. From evolutionary point of view, mitochondria originated from aerobic bacterial endosymbiosis [1]. By the virtue of their own genetic material they are also in some manner autonomous intracellular organelles and sometimes considered organism within organisms. Mitochondria are appropriately called cellular powerhouse as they are major site of ATP production via oxidative phosphorylation. Structurally mitochondria are double walled structures composed of an outer membrane and an inner membrane and their size ranging between ranges from 0.5 to $1.0 \mu \mathrm{m}$ in diameter. Despite its small volume, the intermembrane space between the two membranes is known to house close to 50 proteins (2). It is also involved in transporting macromolecules in and out of the mitochondria, regulating mitochondrial respiration and apoptosis (3). The inner membrane encloses the mitochondrial matrix where most of the soluble proteins of mitochondria, mitochondrial DNA, and transfer RNA are found [4 and references therein].

Given the central role of mitochondria in cellular physiology, any perturbation in its basic functionality leads to catastrophic cellular outcome involving cell death pathways where its dysfunctions play a major role. Given the active role of mitochondria during drug-induced cell death and its relation with cancer chemotherapy, various mitochondrial regulator and pathways of programmed cell death (PCD) are discussed in this review.

\section{Cancer Chemotherapy and Programmed Cell Death}

Chemotherapy for the treatment of cancer was introduced into the clinic more than fifty years ago. Although this form of therapy has been successful for the treatment of some tumors such as testicular cancer and certain leukemias, its success for the treatment of common epithelial tumors of the breast, colon, and lung has been less than spectacular. Ideally, chemotherapeutic drugs should specifically target only neoplastic cells and should decrease tumor burden by inducing cytotoxic and/or cytostatic effects with minimal "collateral damage" to normal cells. In reality, the effectiveness of chemotherapy has suffered from a range of confounding factors including systemic toxicity due to a lack of specificity, rapid drug metabolism, and both intrinsic and acquired drug resistance. [5]. 
It is now clear that some oncogenic mutations disrupt apoptosis, leading to tumor initiation, progression and/or metastasis. Conversely, compelling evidence indicates that other oncogenic changes promote apoptosis, thereby producing selective pressure to override apoptosis during multistage carcinogenesis. It is now well documented that most cytotoxic anticancer agents induce apoptosis raising the intriguing possibility that defects in apoptotic programs contribute to treatment failure. As the same mutations that suppress apoptosis during tumor development also reduce treatment sensitivity, apoptosis provides a conceptual framework to link cancer genetics with cancer therapy [6].

Programmed cell death is a normal physiological process which occurs during embryonic development as well as maintains tissue homeostasis. Although classification of cell death has proven difficult, based on the morphology of dying cells and on the DNA fragmentation or damages, two distinct patterns of cell death have been identified. These have been termed necrosis and apoptosis [7, 8]. PCD is a process, while apoptosis describes a cellular morphology. In most cases, the loss of cells during PCD occurs with apoptotic morphology. Typically, apoptosis involves cellular condensation, genomic DNA fragmentation, deposition of electron dense chromatin along the inner margin of nuclear envelope, formation of membrane blebs that contained portions of nucleus and intact organelles, and ultimately, phagocytosis of the apoptotic bodies by neighboring cells [8]. During chemotherapeutic regime, when a drug targets a cancer cell to its death, apoptotic cell death is a favored cell pathway, which unlike to necrosis does not lad to inflammation and leads to safe disposal of a dead cancer cell.

\section{Mitochondria-Mediated Intrinsic Apoptosis Pathway}

Apoptosis can be triggered in a cell either through the extrinsic pathway or the intrinsic pathway. The extrinsic pathway begins outside a cell, when conditions in the extracellular environment determine that a cell must die. The intrinsic apoptosis pathway begins when an injury occurs within the cell. The intrinsic pathway is initiated through the release of signal factors by mitochondria within the cell so the mitochondrial damage can initiate the intrinsic pathway. A variety of key events focus on mitochondria, including the release of caspase activators, changes in electron transport, loss of mitochondrial transmembrane potential altered cellular oxidation-reduction, and participation of pro- and anti-apoptotic Bcl-2 family proteins. The different signals converge on mitochondria to trigger or inhibit these events and their downstream pathways in physiological cell death [9].

In many apoptosis scenarios, the mitochondrial inner transmembrane potential collapses, indicating the opening of large conductance channel known as the mitochondrial permeability transition (PT) pore. The structure and composition of PT pore remain only partially defined, but its constituents include both inner membrane proteins, such as adenine nucleotide transporter and outer membrane proteins such as porins (voltage dependent anion channel-VDAC), which operate in concert, presumably at inner and outer membrane contact sites, and create a channel through which molecules pass [10]. PT pore opening results in volume dysregulation of mitochondria due to hyperosmolarity of the matrix, which causes the matrix to expand. As the inner membrane with its folded cristae possesses a larger surface area than the outer membrane, this matrix volume expansion can eventually cause outer membrane rupture, releasing apoptogenic proteins [11].

Mitochondria contain several apoptosis inducing proteins in its intermembrane space that can either induce apoptosis involving caspases eg cytochrome c, SMAC, omi/Htra and/or without involving caspases eg AIF, Endonuclease G, Bit 1 etc, and also includes procaspase-2, -3 , and -9 [9]. The apoptotic stimulus induces the release of these proteins into cytosol to activate the pro-caspases present in the cytoplasm. Cytochrome c is the key executioner of apoptosis. A group led by Wang in 1996 demonstrated the role of cytochrome c in initiation of apoptosis for the first time, besides its known involvement in respiration [12]. Following the release of cytochrome $\mathrm{c}$ into the cytoplasm, this molecule binds to the C-terminus of Apa-f1, within the region containing multiple WD-40 motifs. This facilitates the dATP binding to Apaf1 and facilitates the oligomerization of Apaf1, which recruits pro-acapsase-9. This complex formed upon apoptotic stimulus of different proteins is called as apoptosome. In this complex the caspase- 9 is activated and it activates the series of other caspases [13].

Interestingly, apoptosis can also take place independent of caspases. AIF is a protein responsible for caspase-independent chromatin condensation, known as a flavoprotein that is normally confined to inter membrane space of mitochondria [14]. Once apoptosis is induced, AIF translocates from the mitochondrion to the nucleus where it causes partial chromatin condensation and its migration towards the periphery of the nucleus. AIF causes degradation of DNA into fragments greater than $50 \mathrm{~kb}$ in length- a large scale DNA fragmentation that precedes more advanced inter-nucleosomal degradation. In addition to this, Endonuclease $\mathrm{G}$ is another protein that can mediate caspase-independent DNA degradation [15].

SMAC or DABLO (Direct IAP binding protein with low pI) is released into cytosol in cells induced to undergo apoptosis, probably following the exit route of cytochrome c [16]. SMAC/DIABLO binds to inhibitors of apoptosis (IAP) family members and neutralizes their anti-apoptotic activity. Another mitochondrial factor that shares functional properties with SMAC/DIABLO is the serine protease Omi [17] also known as Htra2 [18].

Bit1, a mitochondrial protein is released into the cytoplasm during Loss of cell attachment (anoikis)-induced apoptosis. Cytoplasmic Bit1 forms a complex with AES, a small Groucho/transducin-like enhancer of split (TLE) protein, and induces cell death with characteristics of caspase-independent apoptosis [19]. 


\section{Role of Bcl-2 Family of Proteins During Mitochondria-Mediated Apoptosis}

The Bcl-2 family of proteins constitutes a critical intracellular checkpoint in the mitochondria mediated intrinsic apoptotic pathway featuring both pro-and antiapoptotic members. At least fifteen Bcl-2 family members have been identified in the mammalian cells. The Bcl-2 family can be divided into three main subclasses. All members possess at least one of four domains of sequence similarity, known as Bcl-2 homology (BH) 1-4 domains, roughly corresponding to helices which dictate structure and function [20]. Most prosurvival factors which can inhibit apoptosis contain at least $\mathrm{BH} 1$ and $\mathrm{BH} 2$ and those most similar to Bcl-2 have all four $\mathrm{BH}$ domains. In contrast, proapoptotic members contain the central short (9-16 residues) $\mathrm{BH} 3$ domain.

Bcl-2 was first identified based on its translocation in follicular lymphoma, and is overexpressed in a variety of cancers eg prostate, breast and colon carcinomas, as well as glioblastomas [21]. Bcl-2 supports neoplastic growth, not by stimulating cellular proliferation, but rather by blocking cell death [22]. Moreover Bcl-2 overexpression can accelerate tumorigenesis in transgenic mice, and overexpression of Bcl-2 in transgenic models leads to accumulation of cells due to evasion of normal cell death mechanism [23]. Induction of apoptosis by diverse range of stimuli such as radiation, hyperthermia, growth factor withdrawal glucocorticoid, and multiple classes of chemotherapeutic agents is inhibited by Bcl-2 in in vitro models. The cell death inhibitory effect of Bcl-2 depends upon its expression level, as well as ratio to intracellular levels of proapoptotic Bcl-2 members e.g. Bax. As pro- and antiapoptotic members can bind to each other and seemingly titrate each other's functions, suggesting their relative concentrations may act as a rheostat for the suicide program [10].

Many Bcl-2 family proteins reside in the mitochondria outer membrane, anchored by a hydrophobic stretch of amino acids located within their $-\mathrm{COOH}$ termini, with proteins oriented towards the cytosol. Antiapoptotic Bcl-2 and Bcl-xL suppress the release of caspase activating proteins in the cytosol and maintain the buffered stock of $\mathrm{Ca}+2$ [9]. On the other hand, Bax is a pro-apoptotic Bcl-2-family protein that resides in the cytosol and translocates to mitochondria upon induction of apoptosis [24]. This release is dependent upon induction of the mitochondrial permeability transition, an event that is associated with disruption of the $\Delta \Psi \mathrm{m}[25]$ and has been implicated in a variety of apoptotic phenomena.

The BH3 members e.g. Bad, Bid, Bim, Noxa, Puma etc. serve as upstream sentinels that selectively respond to specific, proximal death and survival signals. For example, the extrinsic pathway is triggered by the engagement of cell surface death receptors, which then activate caspase- 8 that cleaves p22 Bid to connect with the intrinsic death pathway. A reconstituted mitochondrial assay reveals that tBid serve as a membrane-targeted ligand which requires its intact $\mathrm{BH} 3$ domain to trigger oligomerization of Bak or Bax to release cytochrome c [26]. The proapoptotic activity of $\mathrm{BH} 3$ only molecules is apparently kept in check by either transcriptional control in response to DNA damage [10]. Bad is switched on and off by its phosphorylation in response to growth/survival factors [27], providing a connection to the established importance of extracellular factors in promoting cell survival. Activation of $\mathrm{BH} 3$ only molecules both directly or indirectly result in the activation of Bax, Bak and actually require Bax and Bak in executing apoptosis. In contrast, antiapoptotics, such as Bcl-2 and Bcl-xL, serve a principal although perhaps not an exclusive role of binding and sequestering $\mathrm{BH} 3$ only molecules preventing Bax-Bak activation [28].

Exactly how Bid, Bax, and related proteins function to cause cytochrome c release is unclear. In cells given certain apoptotic stimuli, Bid or Bax can translocate to mitochondria [10] to initiate the release of cytochrome c. In some cases, translocation of these proteins may require changes in their conformation [26]. The formation of ion channels in synthetic lipid bilayers by some members of the Bcl-2 family such as Bax, Bcl-xL, and Bcl-2 suggests that proapoptotic members of this family might interact directly with the outer mitochondrial membrane to allow efflux of cytochrome c [11]. Bax has been reported to interact directly with voltage dependent anion channel on the outer membrane to release cytochrome c [29]. Bax may cause instability in artificial lipid membranes, suggesting another mechanism by which Bax may permeabilize the outer mitochondrial membrane [30]. The proapoptotic Bid requires the cooperation of relatives such as Bax for its effect [26]. Bax helps mobilize endoplasmic reticulam $\mathrm{Ca}^{++}$stores, leading to mitochondrial PT, matrix swelling, and rupture of outer mitochondrial membrane. Another possible mechanism is that $\mathrm{BH} 3$ only proteins induce direct outer mitochondrial membrane permeabilization via Bax/Bak-lipid interaction [10].

$\mathrm{Be}$ it the intrinsic and/or pathway of apoptosis, Caspases are the main executioner of apoptosis. Caspase activation is triggered in response to the apoptotic signals and culminates in cleavage of critical proteins, resulting in disassembly of cell. A large body of genetic and biochemical evidences suggest a cascade model for effector caspase activation; a proapoptotic signal culminates in activation of an initiator caspase (e.g. caspase-8, and -9) which, in turn activates effector caspases (caspase-3, and -7), resulting in cellular disassembly [31]. Approximately Forty substrates that have been identified e.g. Poly (ADP) ribose polymerase, b-actin, nuclear lamin protein, gelsolin, caspases, Retinoblastoma, Protein kinase c, DNA-protein kinase, ICAD, IkB, p21, Bcl-2, Bcl-xl, Bid, etc. Nonetheless, the above mentioned proteins play their roles in maintenance of cellular architecture, DNA repair, cell signaling, and apoptosis suggest that a subset of caspases (effectors) is responsible for the cellular changes that occur during apoptosis and provide insight into the mechanisms that they employ [31]. 


\section{Mitochondrial Dysfunction Due to Oxidative Stress}

Reactive oxygen metabolites (ROMs), including superoxide anion $\left(\mathrm{O}_{2} *_{-}\right)$, hydrogen peroxide $(\mathrm{H} 2 \mathrm{O} 2)$ and hydroxyl radical $(* \mathrm{OH})$, play an important role in carcinogenesis. Free redicals, also called reactive species are constantly produced and their physiological concentration is required for normal cell growth and division. An imbalance can lead to dysregulation in cell physiology. Some arise accidentally, an example of which is the leakage of electrons from the mitochondrial electron transport chain to generate superoxide radicals $(\mathrm{O} 2 *-)$, peroxynitrile etc.

An imbalance between cellular production of ROS and the inability of cells to defend against them is called oxidative stress [32]. Multiple antioxidant defense mechanisms exist in a cell e.g. antioxidant enzymes including magnese superoxide dismutase, Copper/Zinc SOD, glutathione peroxidase, and catalase. SODs convert superoxide anions to $\mathrm{H} 2 \mathrm{O} 2$, which is then transformed to $\mathrm{H} 2 \mathrm{O}$ by glutathione peroxidase or by catalase. The damage can become more widespread due to weakened cellular antioxidant defense systems. On the other hand, it was shown that ROS formation depends steeply on $\Delta \Psi \mathrm{m}$ [33]. High protonic potential actuates a mechanism of production of ROS in mitochondria. Hydroxyl radicals are most reactive oxygen free radical species, capable of direct oxidative damage to macromolecules including DNA, protein and lipid membrane. The effect of excess production of ROS such as DNA strand breaks, MPT and subsequently caspase cascade, match the hallmark features of apoptosis. Superoxide dismutase and catalase are important enzymatic defense against oxidative stress that has been shown to be a potentially useful target for anticancer therapy [34]. Oxidative stress results in damage to most of the principal biological macromolecules and in potential apoptotic cell death [35].

\section{Summary}

The focus of this review has been to discuss how mitochondria respond to the stress induced by various cell death inducing agents. From the available literature, it is clear that these cell death inducing agents can induce metabolic stress via ROS generation, MOMP induction or various signaling pathways. There has been conventional cancer chemotherapeutics e.g. cisplastin, dacarbazine, vinblastine etc that can induce programmed cell death. However broader knowledge of targets upstream of mitochondrial apoptotic pathways that direct proapoptotic signaling e.g. BH3 mimetic will provide direct benefits via rationale targeting.

\section{References}

[1] Zimorski, V., Ku, C., Martin, W.F. and Gould, S.B. (2014) Endosymbiotic theory for organelle origins. Curr. Opin. Microbiol., 22C, 38-48.

[2] Vogtle, F.N., Burkhart, J.M., Rao, S., Gerbeth, C., Hinrichs, J.,
Martinou, J.C., Chacinska, A., Sickmann, A., Zahedi, R.P. and Meisinger, C. (2012) Intermembrane space proteome of yeast mitochondria. Mol. Cell. Proteomics, 11, 1840-1852.

[3] Herrmann, J.M., Longen, S., Weckbecker, D. and Depuydt, M. (2012) Biogenesis of mitochondrial proteins. Adv. Exp. Med. Biol., 748, 41-64.

[4] Kim A. A panoramic overview of mitochondria and mitochondrial redox biology. Toxicol Res. 2014 Dec; 30(4): 221-34. doi: 10.5487/TR.2014.30.4.221

[5] Johnstone RW, Ruefli AA, Lowe SW (2002) Apoptosis: a link between cancer genetics and chemotherapy. Cell 108: 153-64.

[6] Lowe SW, Lin AW (2000) Apoptosis in cancer. Carcinogenesis 21: 485-95.

[7] Kerr JF, Wyllie AH, Currie AR (1972) Apoptosis: a basic biological phenomenon with wide-ranging implications in tissue kinetics. Br J Cancer 26: 239-57.

[8] Majno G, Joris I (1995) Apoptosis, oncosis, and necrosis. An overview of cell death. Am J Pathol 146: 3-15.

[9] Green DR, Reed JC (1998) Mitochondria and apoptosis. Science 281: 1309-12.

[10] Danial NN, Korsmeyer SJ (2004) Cell death: critical control points. Cell 116: 205-19.

[11] Newmeyer DD, Ferguson-Miller S (2003) Mitochondria: releasing power for life and unleashing the machineries of death. Cell 112:481-90.

[12] Liu X, Kim CN, Yang J, Jemmerson R, Wang X (1996) Induction of apoptotic program in cell-free extracts: requirement for dATP and cytochrome c. Cell 86: 147-57.

[13] Adrain C, Martin SJ (2001) The mitochondrial apoptosome: a killer unleashed by the cytochrome seas. Trends Biochem Sci 26: 390-7.

[14] Lorenzo HK, Susin SA, Penninger J, Kroemer G (1999) Apoptosis inducing factor (AIF): a phylogenetically old, caspase-independent effector of cell death. Cell Death Differ 6:516-24.

[15] van Loo G, Schotte P, van Gurp M, Demol H, Hoorelbeke B, Gevaert K, Rodriguez I, Ruiz-Carrillo A, Vandekerckhove J, Declercq W, Beyaert R, Vandenabeele P. (2001) Endonuclease $\mathrm{G}$ : a mitochondrial protein released in apoptosis and involved in caspase-independent DNA degradation. Cell Death Differ 8: $1136-42$.

[16] Du C, Fang M, Li Y, Li L, Wang X (2000) Smac, a mitochondrial protein that promotes cytochrome c-dependent caspase activation by eliminating IAP inhibition. Cell 102: $33-42$

[17] Faccio L, Fusco C, Chen A, Martinotti S, Bonventre JV, Zervos AS (2000) Characterization of a novel human serine protease that has extensive homology to bacterial heat shock endoprotease HtrA and is regulated by kidney ischemia. J Biol Chem 275: 2581-8.

[18] Gray CW, Ward RV, Karran E, Turconi S, Rowles A, Viglienghi D, Southan C, Barton A, Fantom KG, West A, Savopoulos J, Hassan NJ, Clinkenbeard H, Hanning C, Amegadzie B, Davis JB, Dingwall C, Livi GP, Creasy CL (2000) Characterization of human HtrA2, a novel serine protease involved in the mammalian cellular stress response. Eur J Biochem 267: $5699-710$ 
[19] Jan Y, Matter M, Pai JT, Chen YL, Pilch J, Komatsu M, Ong E, Fukuda M, Ruoslahti E (2004) A mitochondrial protein, Bit1, mediates apoptosis regulated by integrins and Groucho/TLE corepressors. Cell 116: 751-62.

[20] Adams JM, Cory S (1998) The Bcl-2 protein family: arbiters of cell survival. Science 281:1322-6.

[21] Reed JC (1999) Dysregulation of apoptosis in cancer. J Clin Oncol 17: 2941-53.

[22] Hockenbery D, Nunez G, Milliman C, Schreiber RD, Korsmeyer SJ (1990) Bcl-2 is an inner mitochondrial membrane protein that blocks programmed cell death. Nature 348: $334-6$

[23] McDonnell TJ, Deane N, Platt FM, Nunez G, Jaeger U, McKearn JP, Korsmeyer SJ (1989) bcl-2-immunoglobulin transgenic mice demonstrate extended $\mathrm{B}$ cell survival and follicular lymphoproliferation. Cell 57: 79-88.

[24] Hsu YT, Wolter KG, Youle RJ (1997) Cytosol-to-membrane redistribution of $\mathrm{Bax}$ and $\mathrm{Bcl}-\mathrm{X}(\mathrm{L})$ during apoptosis. Proc Natl Acad Sci USA 94: 3668-72.

[25] Pastorino JG, Chen ST, Tafani M, Snyder JW, Farber JL (1998) The overexpression of Bax produces cell death upon induction of the mitochondrial permeability transition. J Biol Chem 273: 7770-5.

[26] Desagher S, Osen-Sand A, Nichols A, Eskes R, Montessuit S, Lauper S, Maundrell K, Antonsson B, Martinou JC (1999) Bid-induced conformational change of Bax is responsible for mitochondrial cytochrome $\mathrm{c}$ release during apoptosis. J Cell Biol 144: 891-901.

[27] Zha J, Harada H, Yang E, Jockel J, Korsmeyer SJ (1996) Serine phosphorylation of death agonist BAD in response to survival factor results in binding to $14-3-3$ not BCL-X(L) Cell 87: 619-28.

[28] Cheng EH, Wei MC, Weiler S, Flavell RA, Mak TW, Lindsten T, Korsmeyer SJ (2001) BCL-2, BCL-X(L) sequester BH3 domain-only molecules preventing $\mathrm{BAX}$ - and BAK-mediated mitochondrial apoptosis. Mol Cell 8:705-11.

[29] Narita M, Shimizu S, Ito T, Chittenden T, Lutz RJ, Matsuda H, Tsujimoto Y (1998) Bax interacts with the permeability transition pore to induce permeability transition and cytochrome c release in isolated mitochondria. Proc Natl Acad Sci USA 95: 14681-6.

[30] Basanez G, Nechushtan A, Drozhinin O, Chanturiya A, Choe E, Tutt S, Wood KA, Hsu Y, Zimmerberg J, Youle RJ (1999) Bax, but not Bcl-xL, decreases the lifetime of planar phospholipid bilayer membranes at subnanomolar concentrations. Proc Natl Acad Sci U S A 96: 5492-7.

[31] Thornberry NA, Lazebnik Y (1998) Caspases: enemies within. Science 281: 1312-6.

[32] Simonian NA, Coyle JT (1996) Oxidative stress in neurodegenerative diseases. Annu Rev Pharmacol Toxicol 36: 83-106.

[33] Korshunov SS, Skulachev VP, Starkov AA. (1997) High protonic potential actuates a mechanism of production of reactive oxygen species in mitochondria. FEBS Lett 416:15-8.

[34] Huang P, Feng L, Oldham EA, Keating MJ, Plunkett W (2000) Superoxide dismutase as a target for the selective killing of cancer cells. Nature 407(6802):390-5.

[35] Chandra J, Samali A, Orrenius S (2000) Triggering and modulation of apoptosis by oxidative stress. Free Radic Biol Med 29:323-33. 